\title{
Brittle Layer Cracking on Bonded Loaded Substrate
}

\author{
K. P. Mróz and K. Doliński
}

\begin{abstract}
A bi-material element composed of two plates bonded by an interface and subjected to monotonically increasing loading is considered. The cohesive zone model is assumed to simulate the interface response in shear. The coating layer response is characterized by the critical failure stress. The layer cracking modes are analyzed by applying the shear lag approach. The analytical solution illustrates the effect of strength on cracking of the coating layer.
\end{abstract}

Index Terms - Fragmentation, cohesive zone model, shear lag model, mechanical loading.

\section{INTRODUCTION}

The damage of layers or coatings on the substrate are one of the most important problem occurring in this type of composite materials. In most cases the delamination and cracking are the dominant modes of degradation of materials performances. Although the coatings are usually designed to improve surface tribological properties it should be noted that deposition technique can also induce the negative aspects of the strength of the material, especially exhibited during cyclic loading [1]. Much effort has been invested over the last years to research in this area. However, the mechanical models are often complicated and not able to fully explain the failure mechanisms. Recently, the concept of cohesive zones assumed ahead of crack tip has been applied, cf. Białas and Mróz [2], [3], becoming a powerful analytical tool in the nonlinear fracture analysis of progressive delamination and cracking of thin layer bonded to a rigid or elastic substrate and subjected to thermal loading. Similar boundary conditions were considered by Nikolova et al. [4]. In the last decades the shear lag approach is one of the most used tools in mechanics of composite materials, where stress transfer occurs via an interfacial shear stress. This idea was introduced by Cox [5] as a simple one-dimensional equation for analysing stress transfer between a fiber and matrix assuming a fully elastic interface, and by Kelly and Tyson [6] for a fully plastic interface. The main idea of the shear-lag analysis is an assumption which involves in-plane shear stress $\tau_{x y}$ and decouples the 2D plane problem into two 1D ones. Hedgepeth [7] was the first who applied shear-lag model to unidirectional composites. Recently, the bi-material structure consisting of two elastic isotropic plates bonded together subject to mechanical tension was studied by Ivanova et al. [8]. The phenomenological model describing cracking under uniaxial tensile strain of brittle thin film on a deformable substrate with an elastic-plastic interface layer was considered by McGuigan

Manuscript received April 27, 2015; revised July 24, 2015.

The authors are with the Institute of Fundamental Technological Research, Polish Academy of Science, Pawińskiego 5b, 02-106 Warsawa, Poland (e-mail: kmroz@ ippt.pan.pl,kdolin@ ippt.pan.pl). et al. [9]. They presented the analytical solution predicting averaged crack density as a function of applied strain and materials parameters and showed good correlation with experimental data.

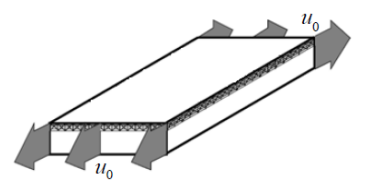

a) b)
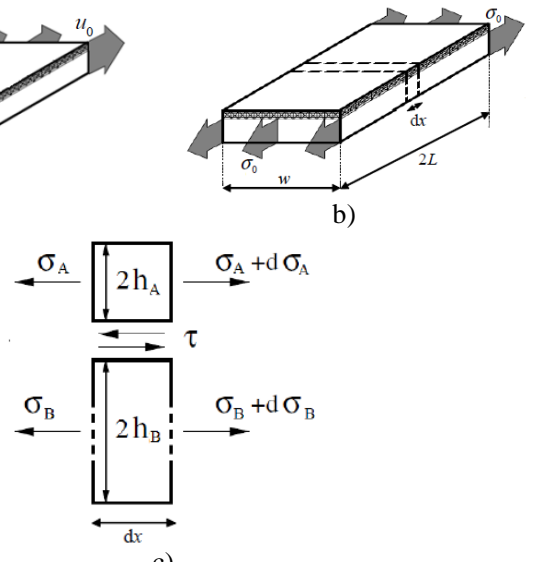

c)

Fig. 1. a) Composites loading by displacement b) Substrate loading c) Stress acting on an infinitesimal bi-layer element.

\section{PROBLEM FORMULATION}

Consider a plate of length $2 L$ and two plates A and B of different materials and thicknesses, $2 h_{\mathrm{A}} ; 2 h_{\mathrm{B}}$, respectively. The plates are bonded together along the interface I and mechanical loaded in the monotonic way. It is assumed that the layers and interface are of isotropic materials, the bending effects are neglected and the axial stresses and strains are uniform over the cross sections of the plates. The both components of the consider composite are working only on tension-pressure, while the interface works on the shear, only. The main interest of this study is to determine the damage response of the consider structure to the different monotonic mechanical loading and compare it with the possible design variables.

The following differential equations (Fig. 1) of plate equilibrium can be formulated

$$
\frac{d \sigma_{A}}{d x}=\frac{\tau_{I}}{2 h_{A}}, \quad \frac{d \sigma_{B}}{d x}=-\frac{\tau_{I}}{2 h_{B}}
$$

\section{Constitutive Models}

The simplified constitutive law for the interface layer and the coatings is assumed as shown in Fig. 2. The substrate is assumed to be full elastic. The presented law is divided into two parts. The first one is the elastic part, and the second one can be considered in various ways for the material behaviours:

- brittle behavior,

- plastic weakness,

- ductile behavior. 
a)

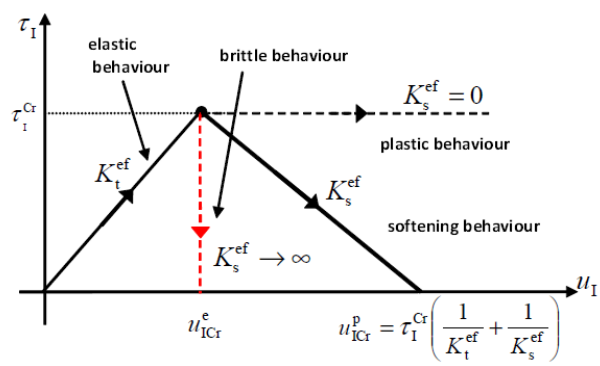

b)

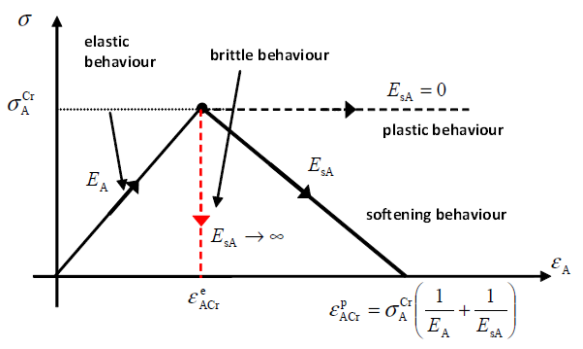

Fig. 2. The interface and the coating models a), b) respectively.

For the elastic part we have the interface response as follows

$$
\tau_{I}^{e}(x)=K_{t} \gamma=\frac{K_{t}}{h} u_{I}^{e}=K_{t}^{e f} u_{I}^{e}
$$

where,

$$
\gamma=\frac{u_{A}^{e}-u_{B}^{e}}{h}=\frac{u_{I}}{h} .
$$

Here the elastic shear modulus is denoted by $K_{t}$. The thickness $h$ of the bonding layer occurs only in the effective shear modulus $K_{t}^{e f}=K_{t} / h$. However, it is convenient to introduce the non-dimensional displacement discontinuity $\bar{u}_{I}^{e}=u_{I}^{e} /\left(h_{A}+h_{B}\right)$. Then the relation (2) can be expressed as follows

$$
\tau_{I}^{e}(x)=\frac{K_{w}}{h_{A}+h_{B}} u_{I}^{e}=K_{w} \bar{u}_{I}^{e}
$$

where, $K_{w}=K_{t}\left(h_{A}+h_{B}\right) / h=K_{t}^{e f}\left(h_{A}+h_{B}\right)$. For specified loading when the stress in the coating reaches its maximum critical value $\sigma_{A}^{C r}$ the three different behavior can occur in Fig. 2b). In the article the elastic-brittle situation is only assumed, when the fracture process begins immediately after the tension strength is attained.

\section{SUBSTRATE LOADING}

Let's consider the bi-layer with two alternative monotonic loading conditions, namely the tensile displacement $u_{0}$ or the stress $\sigma_{0}$ acting on the border of the element in Fig. 3a).

In the elastic case with non-dimensional variables the following forms can be assumed

$$
\begin{aligned}
& \left(h_{A}+h_{B}\right) \bar{x}=x,\left(h_{A}+h_{B}\right) \bar{u}_{i}^{e}=u_{i}^{e}, \xi=\frac{h_{A}}{h_{B}}, \eta=\frac{E_{A}}{E_{B}}, \\
& \bar{\varepsilon}_{i}^{e}=\varepsilon_{i}^{e}, E_{B} \bar{\sigma}_{i}^{e}=\sigma_{i}^{e}, E_{B} \bar{\tau}_{i}^{e}=\tau_{i}^{e}, \bar{K}_{t}=K_{w} / E_{B}, \\
& \bar{K}_{t}^{e f}=\bar{K}_{t} E_{B} /\left(h_{A}+h_{B}\right)
\end{aligned}
$$

and for the boundary conditions

$$
\bar{u}_{A}^{e}(0)=0, \bar{u}_{B}^{e}(0)=0, \bar{\sigma}_{A}^{e}(\bar{L})=0
$$

and

$$
\begin{array}{ll}
\bar{\sigma}_{B}^{e}(\bar{L})=\bar{\sigma}_{0} & \text { for stress boundary condition, } \\
\bar{u}_{B}^{e}(\bar{L})=\bar{u}_{0} & \text { for displacement boundary condition. }
\end{array}
$$

Then Eq. (1) becomes:

$$
\frac{d \bar{\sigma}_{A}}{d \bar{x}}=\bar{\tau}_{I} \frac{1+\xi}{2 \xi}, \frac{d \bar{\sigma}_{B}}{d \bar{x}}=-\bar{\tau}_{I} \frac{1+\xi}{2}
$$

Subsequently, the Eq. (6) can be expressed in the following form

$$
\frac{d \bar{\sigma}_{A}}{d \bar{x}}=\frac{\lambda^{2}}{1+\eta \xi} \bar{u}_{I}^{e}, \frac{d \bar{\sigma}_{B}}{d \bar{x}}=-\frac{\lambda^{2}}{1+\eta \xi} \eta \xi \bar{u}_{I}^{e}
$$

where

$$
\lambda^{2}=\frac{\bar{K}_{t}(1+\eta)(1+\eta \xi)}{2 \eta \xi} .
$$

Simultaneously, the following equations can be formulated

\begin{tabular}{|c|c|}
\hline $\bar{\sigma}_{0}$ & $\bar{u}_{0}$ \\
\hline $\bar{u}_{\mathrm{A}}^{\mathrm{e}}(\bar{x})=\bar{\sigma}_{0} \frac{\bar{x} \bar{\lambda}_{\mathrm{e}}-\operatorname{sech}\left[\bar{\lambda}_{\mathrm{e}} L\right] \sinh \left[\bar{x} \bar{\lambda}_{\mathrm{e}}\right]}{\bar{\lambda}_{\mathrm{e}}+\bar{\lambda}_{\mathrm{e}} \eta \xi}$ & $\bar{u}_{\mathrm{A}}^{\mathrm{e}}(\bar{x})=\bar{u}_{0} \frac{\bar{x} \lambda \cosh \left[L \bar{\lambda}_{\mathrm{e}}\right]-\sinh \left[\bar{x} \bar{\lambda}_{\mathrm{e}}\right]}{L \bar{\lambda}_{\mathrm{e}} \cosh \left[L \overline{\mathrm{e}}_{\mathrm{e}}\right]+\eta \xi \sinh \left[L \bar{\lambda}_{\mathrm{e}}\right]}$ \\
\hline $\bar{u}_{\mathrm{B}}^{\mathrm{e}}(\bar{x})=\bar{\sigma}_{0} \frac{\bar{x} \bar{\lambda}_{\mathrm{e}}+\eta \xi \operatorname{sech}\left[\bar{\lambda}_{\bar{L}} \bar{L}\right] \sinh \left[\bar{x} \bar{\lambda}_{\mathrm{e}}\right]}{\bar{\lambda}_{\mathrm{e}}+\bar{\lambda}_{\mathrm{e}} \eta \xi}$ & $\bar{u}_{\mathrm{B}}^{\mathrm{e}}(\bar{x})=\bar{u}_{0} \frac{\bar{x} \bar{\lambda} \cosh \left[\bar{L} \bar{\lambda}_{\mathrm{e}}\right]+\eta \xi \sinh \left[\bar{x} \bar{\lambda}_{\mathrm{e}}\right]}{\bar{L} \bar{\lambda}_{\mathrm{e}} \cosh \left[\bar{L}_{\mathrm{e}}\right]+\eta \xi \sinh \left[\bar{L} \bar{\lambda}_{\mathrm{e}}\right]}$ \\
\hline $\bar{u}_{1}^{\mathrm{e}}(\bar{x})=-\bar{\sigma}_{0} \frac{\operatorname{sech}\left[L \bar{\lambda}_{e}\right] \sinh \left[\bar{x} \bar{\lambda}_{e}\right]}{\bar{\lambda}_{e}}$ & $\bar{u}_{1}^{\mathrm{e}}(\bar{x})=-\bar{u}_{0} \frac{(1+\eta \xi) \sinh \left[\bar{x} \bar{\lambda}_{\mathrm{e}}\right]}{\bar{L} \bar{\lambda}_{\mathrm{e}} \cosh \left[L \bar{\lambda}_{\mathrm{e}}\right]+\eta \xi \sinh \left[L \bar{\lambda}_{\mathrm{e}}\right]}$ \\
\hline $\bar{\varepsilon}_{\mathrm{A}}^{\mathrm{e}}(\bar{x})=\bar{\sigma}_{0} \frac{1-\cosh \left[\bar{x} \bar{\lambda}_{\mathrm{e}}\right] \operatorname{sech}\left[L \bar{\lambda}_{\mathrm{e}}\right]}{1+\eta \xi}$ & $\bar{\varepsilon}_{\mathrm{A}}^{\mathrm{e}}(\bar{x})=\bar{u}_{0} \bar{\lambda} \frac{-\cosh \left[\bar{x} \bar{\lambda}_{\mathrm{e}}\right]+\cosh \left[\bar{L} \bar{\lambda}_{\mathrm{e}}\right]}{\bar{L} \bar{\lambda}_{\mathrm{e}} \cosh \left[\bar{L} \bar{\lambda}_{\mathrm{e}}\right]+\eta \xi \sinh \left[\bar{L} \bar{\lambda}_{\mathrm{e}}\right]}$ \\
\hline $\bar{\varepsilon}_{\mathrm{B}}^{\mathrm{e}}(\bar{x})=\bar{\sigma}_{0} \frac{1+\eta \xi \cosh \left[\bar{x} \bar{x}_{\mathrm{e}}\right] \operatorname{sech}\left[L \bar{\lambda}_{\mathrm{e}}\right]}{1+\eta \xi}$ & $\bar{\varepsilon}_{\mathrm{B}}^{\mathrm{e}}(\bar{x})=\bar{u}_{0} \bar{\lambda} \frac{\eta \xi \cosh \left[\bar{x} \bar{\lambda}_{\mathrm{e}}\right]+\cosh \left[L \bar{\lambda}_{\mathrm{e}}\right]}{\bar{L} \bar{\lambda}_{\mathrm{e}} \cosh \left[\bar{L} \bar{\lambda}_{\mathrm{e}}\right]+\eta \xi \sinh \left[L \bar{\lambda}_{\mathrm{e}}\right]}$ \\
\hline $\bar{\sigma}_{\mathrm{A}}^{\mathrm{e}}(\bar{x})=\bar{\sigma}_{0} \eta\left(\frac{1-\cosh \left[\bar{x}_{\mathrm{e}}\right] \operatorname{sech}\left[L \bar{\lambda}_{\mathrm{e}}\right]}{1+\eta \xi}\right)$ & $\bar{\sigma}_{\mathrm{A}}^{\mathrm{e}}(\bar{x})=\eta \bar{u}_{0} \bar{\lambda} \frac{-\cosh \left[\bar{x} \bar{\lambda}_{\mathrm{e}}\right]+\cosh \left[\bar{L} \bar{\lambda}_{\mathrm{e}}\right]}{\bar{L} \bar{\lambda}_{\mathrm{e}} \cosh \left[\bar{L} \overline{\mathrm{e}}_{\mathrm{e}}\right]+\eta \xi \sinh \left[\mid \bar{\lambda}_{\mathrm{e}}\right]}$ \\
\hline $\bar{\sigma}_{\mathrm{B}}^{\mathrm{e}}(\bar{x})=\bar{\sigma}_{0} \frac{1+\eta \xi \cosh \left[\bar{x}_{\mathrm{e}}\right] \operatorname{sech}\left[L \bar{\lambda}_{\mathrm{e}}\right]}{1+\eta \xi}$ & $\bar{\sigma}_{\mathrm{B}}^{\mathrm{e}}(\bar{x})=\bar{u}_{0} \bar{\lambda} \frac{\eta \xi \cosh \left[\bar{\lambda}_{\mathrm{e}}\right]+\cosh \left[L \bar{\lambda}_{\mathrm{e}}\right]}{\bar{L} \bar{\lambda}_{\mathrm{e}} \cosh \left[\bar{L} \bar{\lambda}_{\mathrm{e}}\right]+\eta \xi \sinh \left[\bar{L} \bar{\lambda}_{\mathrm{e}}\right]}$ \\
\hline $\bar{\tau}_{\mathrm{I}}^{\mathrm{e}}(\bar{x})=-\bar{K}_{\mathrm{t}} \bar{\sigma}_{0}\left(\frac{\operatorname{sech}\left[L \bar{\lambda}_{\mathrm{e}}\right] \sinh \left[\bar{x} \bar{\lambda}_{\mathrm{e}}\right]}{\bar{\lambda}_{\mathrm{e}}}\right)$ & $\bar{\tau}_{\mathrm{I}}^{\mathrm{e}}(\bar{x})=-\bar{K}_{\mathrm{t}} \bar{u}_{0} \frac{(1+\eta \xi) \sinh \left[\bar{x} \bar{\lambda}_{\mathrm{e}}\right]}{\bar{L} \bar{\lambda}_{\mathrm{e}} \cosh \left[\bar{L} \bar{\lambda}_{\mathrm{e}}\right]+\eta \xi \sinh \left[\overline{\lambda_{\mathrm{e}}}\right]}$ \\
\hline
\end{tabular}

$$
\frac{d^{2} \bar{u}_{I}^{e}}{d \bar{x}^{2}}=\lambda^{2} \bar{u}_{I}^{e}
$$

and

$$
\frac{d^{2} \bar{u}_{A}^{e}}{d \bar{x}^{2}}=\frac{\lambda^{2}}{1+\eta \xi} \bar{u}_{I}^{e}, \frac{d^{2} \bar{u}_{B}^{e}}{d \bar{x}^{2}}=-\frac{\lambda^{2}}{1+\eta \xi} \eta \xi \bar{u}_{I}^{e}
$$

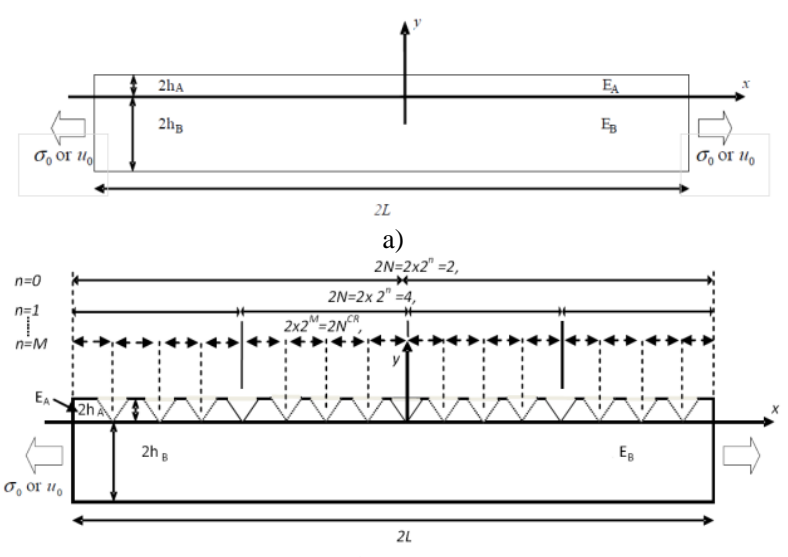

b)

Fig. 3. a) The bi-layer mechanical loading. b) Fragmentation process.

TABLE I: THE EXPRESSION FOR ELASTIC STRESS, STRAIN AND DISPLACEMENTS IN DIMENSIONLESS PARAMETERS FOR SUBSTRATE LOADING AND NOT CRACKED LAYER A 
To find the mechanical fields the Eq. (9) with the boundary conditions should be solved, as presented in Table I. Fig. 4a) shows the example of the mechanical field distributions for not cracked bi-layer under stress tensile conditions.

Let us now consider a brittle fracture of the layer A with $\bar{\sigma}_{A}^{C}$ indicating the strength in tension. As it is seen in Fig. 4a) the maximum value of normal stress is attained along the entire middle zone.
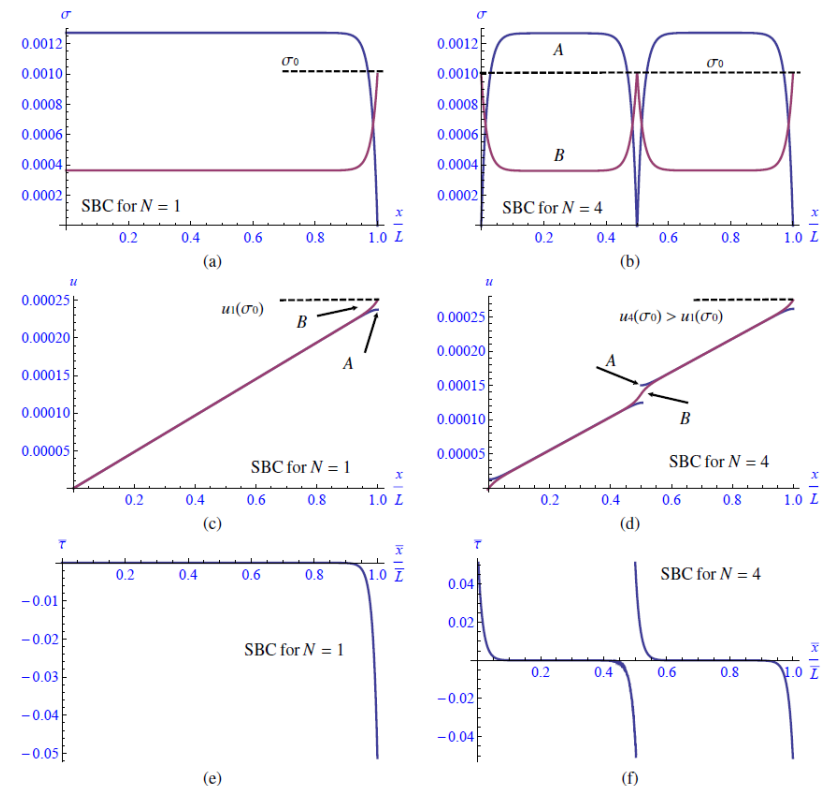

Fig. 4. The stress fields under stress condition, SBC for (a) one segment (b) four segments. The displacement fields for (c) one segment (d) four segments. The shear stress fields for (e) one segment (f) four segments.

TABLE II: THE EXPRESSION FOR ELASTIC STRESS, STRAIN AND DISPLACEMENTS IN DIMENSIONLESS PARAMETERS FOR SUBSTRATE LOADING AND 2N SEGMENTS

\begin{tabular}{|c|c|}
\hline $\bar{\sigma}_{0}$ & $\bar{u}_{0}$ \\
\hline 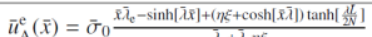 & 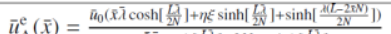 \\
\hline 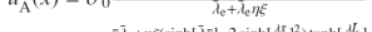 & $u_{\mathrm{A}}^{\prime}(x)=\frac{L \bar{i} \cosh \left[\frac{L}{2 N}\right]+2 N \eta \xi \sinh \left[\frac{L}{2 N}\right]}{2 N}$ \\
\hline 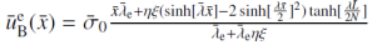 & 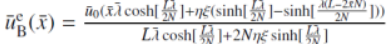 \\
\hline$-\sinh \left[\bar{x}_{\mathrm{e}}\right]+\cosh \left[\bar{x}_{\mathrm{e}}\right] \tanh \left[\frac{\lambda T}{2 N}\right]$ & $(1+\eta E) \sinh \left[\frac{i(L-2 \pi N)}{2 N}\right]$ \\
\hline & 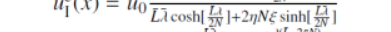 \\
\hline $\bar{\varepsilon}_{\mathrm{A}}^{\mathrm{e}}(\bar{x})=\bar{\sigma}_{0} \frac{1-\cosh \left[\bar{x}_{\mathrm{e}}\right]+\sinh \left[\bar{x}_{\mathrm{e}}\right] \tanh \left[\frac{\lambda I}{2 \nu}\right]}{1+\eta \xi}$ & 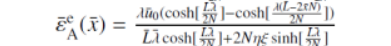 \\
\hline $\bar{\varepsilon}_{0}^{\mathrm{e}}(\bar{x})=\bar{\sigma}_{0} \frac{1+\eta \xi\left(\cosh \left[\bar{x}_{e}\right]-\sinh \left[\bar{x}_{e}\right] \tanh \left[\frac{\lambda}{2 \Delta}\right]\right)}{L}$ & 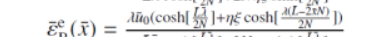 \\
\hline 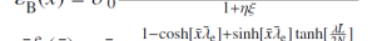 & 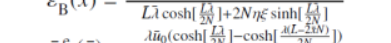 \\
\hline $\bar{\sigma}_{\mathrm{A}}^{\mathrm{e}}(\bar{x})=\bar{\sigma}_{0} \eta \underline{1-\cos \mid}$ & 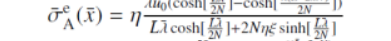 \\
\hline $\bar{\sigma}_{\mathrm{B}}^{\mathrm{e}}(\bar{x})=\bar{\sigma}_{0} \frac{1+\eta \xi\left(\cosh \left[x_{\bar{e}}\right]-\sinh \left[x \bar{\lambda}_{\mathrm{e}}\right] \tanh \left[\frac{{ }^{2}}{2 \nu}\right]\right)}{1+\eta \xi}$ & 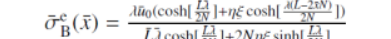 \\
\hline $\bar{\tau}_{1}^{\mathrm{e}}(\bar{x})=\bar{K}_{\mathrm{t}} \bar{\sigma}_{0} \frac{-\sinh \left[\bar{x}_{e}\right]+\cosh \left[\bar{x}_{e}\right] \tanh \left[\frac{\mathcal{L}}{2 \pi}\right]}{\bar{\lambda}}$ & 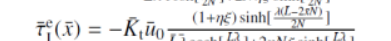 \\
\hline T(n) $=000$ & ${ }^{0} \overline{L i} \cosh \left[\frac{L_{2}}{2 N}\right]+2 \eta N \xi \sinh \left[\frac{L_{2}}{2 N}\right]$ \\
\hline
\end{tabular}

So, it is assumed that this is the place where the fracture occurs. It means that the maximum stress is always in the middle of each coating's fragment. Then, a new boundary condition is created and the stress field has to satisfy there the condition $\bar{\sigma}_{A}^{e}(0)=0$. The fragmentation process continues until no more critical stresses $\bar{\sigma}_{A}^{C}$ under a given loading are attained at any fragments of the plate $\mathrm{A}$.

Thus, the total number of fragments $2 N^{C R}$ may equal $2 N=2 \cdot 2^{n}$, where $n \geq 0$ according to Fig. $3 b$ ). The length of each fragment has been used with the non-dimensional stress boundary conditions (SBC) as

$$
\bar{\sigma}_{A}^{e}(0)=0, \bar{u}_{B}^{e}(0)=0, \bar{\sigma}_{A}^{e}(\bar{L} / N)=0, \bar{\sigma}_{B}^{e}(\bar{L} / N)=\bar{\sigma}_{0}
$$

To find the mechanical fields the Eq. (9) with the above boundary conditions should be also solved for displacement boundary condition (DBC) as presented in Table II, when $\bar{u}_{B}^{e}(\bar{L} / N)=\bar{u}_{0} / N$ is applied. Fig. $\left.4 \mathrm{~b}\right)$ shows the example of the mechanical field distributions for four segments of the layer A under stress tensile conditions.

Then the condition that the maximal value of stress in the middle of each coating's fragment can be formulated for SBC as

$$
\bar{\sigma}_{A}^{e}\left(\frac{\bar{L}}{2 N}\right)=\bar{\sigma}_{0} \eta \frac{1-\cosh \left[\frac{\bar{L}}{2 N} \bar{\lambda}_{e}\right]+\sinh \left[\frac{\bar{L} \bar{\lambda}_{e}}{2 N}\right] \tanh \left[\frac{\bar{L} \bar{\lambda}_{e}}{2 N}\right]}{1+\eta \xi} \geq \bar{\sigma}_{A}^{C}
$$

and for DBC as

$$
\bar{\sigma}_{A}^{e}\left(\frac{\bar{L}}{2 N}\right)=\bar{u}_{0} \eta \bar{\lambda}_{e} \frac{\cosh \left[\frac{\bar{L}}{2 N} \bar{\lambda}_{e}\right]-1}{\bar{L} \bar{\lambda}_{e} \cosh \left[\frac{\bar{L}}{2 N}\right]+2 N \eta \xi \sinh \left[\frac{\bar{L}}{2 N}\right]} \geq \bar{\sigma}_{A}^{C}
$$

Then the total number of the fragments is the smallest natural number $2 N$ obtained for the applied stress, $\bar{\sigma}_{0}$, as
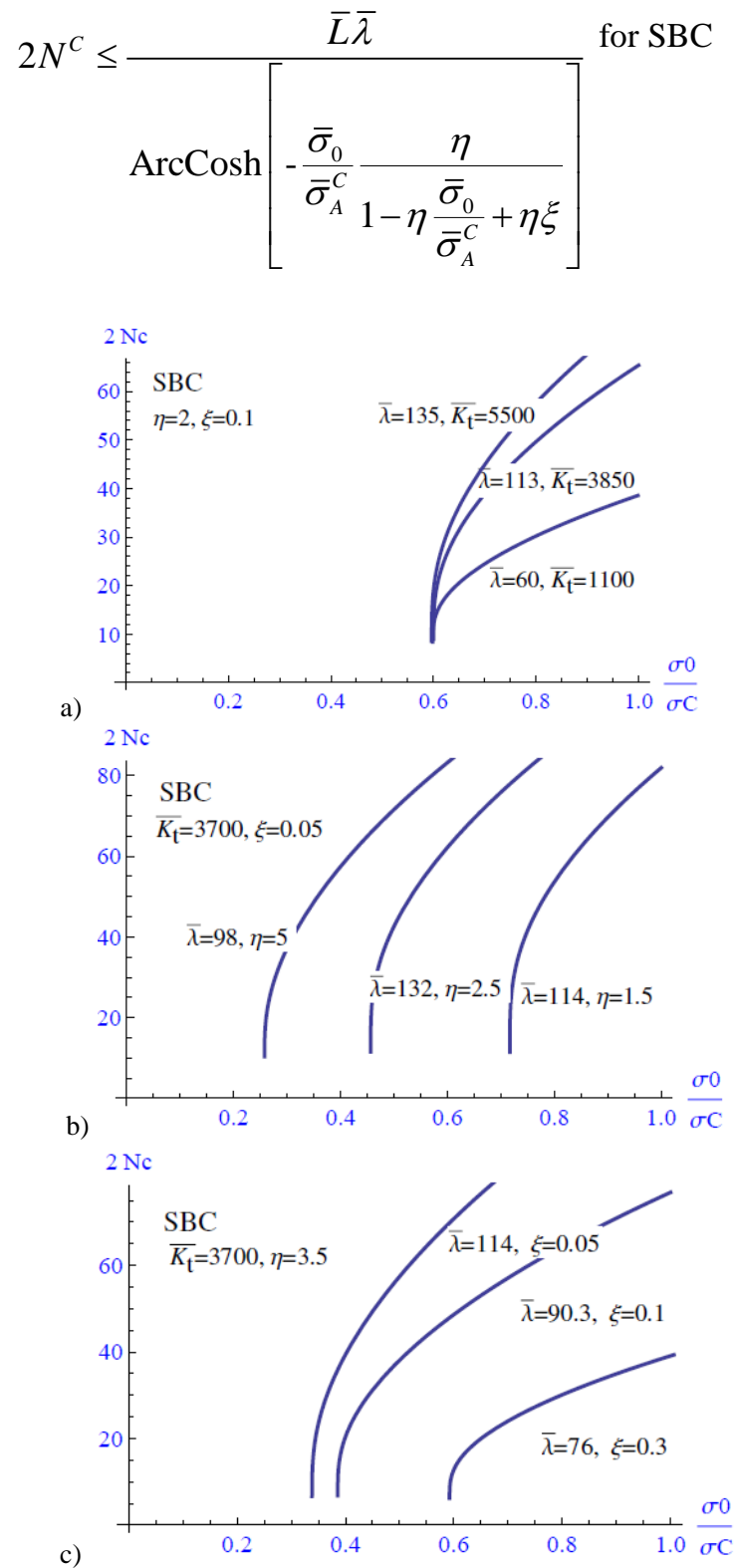


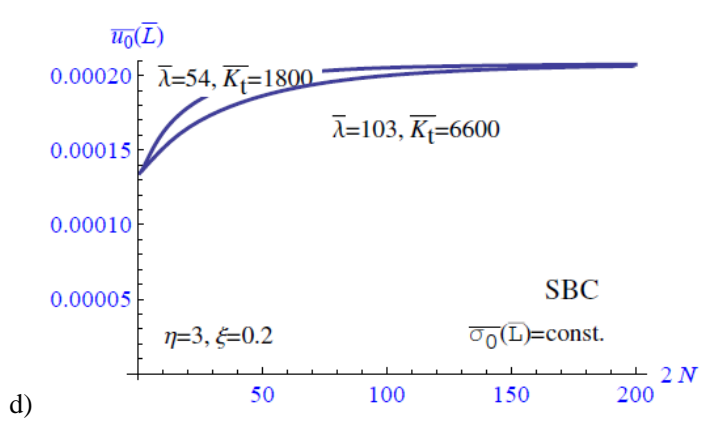

Fig. 5. a)-c) Dependence between the maximal number of fragments $2 N^{C}$ and applied stress. d) Change of the displacement on the corner specimen $\bar{x}=\bar{L}$ in the layer B after segmentation process under SBC.

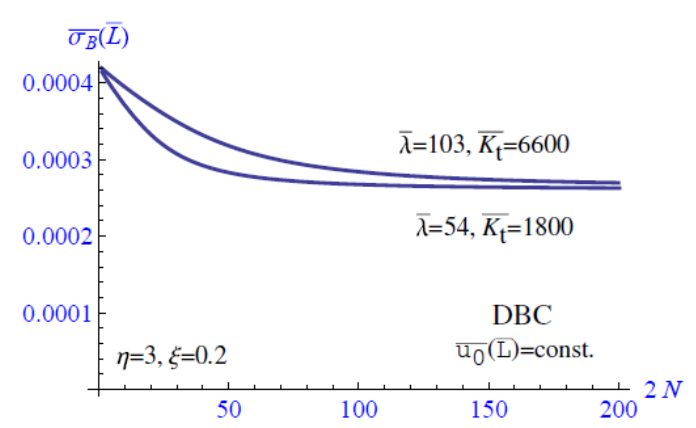

Fig. 6. Change of the stress on the corner specimen $\bar{x}=\bar{L}$ in the layer B after segmentation process under DBC.

However, for DBC Eq. (10b) should be resolved numerically.

Fig. 5a)-Fig. 5c) presents the total possible number of the coating fragments, $2 N$, depending on the loading parameter $\bar{\sigma}_{0} / \bar{\sigma}_{C}^{A}$ for different material data. Fig. $5 \mathrm{~d}$ ) presents the change of the displacement on the corner specimen $\bar{x}=\bar{L}$ in the layer B after segmentation process under SBC. Let us note that the displacement is growing with growth of the segmentation process. It means that stiffness of the layer A decreases due to the cracking process.

Fig. 6 presents the change of the stress on the corner specimen $\bar{x}=\bar{L}$ in the layer $\mathrm{B}$ after segmentation process under DBC. Let us note that the stress is decreasing with developing of the segmentation process. It means that stiffness of the layer A decreases also due to the cracking process.

Based on the Eq. (10a) the initial relation $F=\bar{\sigma}_{0}^{\text {Init }} / \bar{\sigma}_{A}^{C}$ for beginning the fragmentation process (for $N=1$ and assumed that fragmentation is prevailing process) can be obtained for $\mathrm{SBC}$ as

$$
F=\frac{\bar{\sigma}_{0}^{\text {Init }}}{\bar{\sigma}_{A}^{C}}=\frac{1+\eta \xi}{2 \eta} \frac{1}{2+\operatorname{csch}\left[\frac{\bar{L} \overline{\lambda_{e}}}{4}\right]^{2}}
$$

and for DBC on the Eq. (10b)

$$
F=\frac{\bar{u}_{0}^{\text {Init }}}{\bar{\sigma}_{A}^{C}}=\frac{\bar{L} \bar{\lambda}_{e}+4 \eta \xi \operatorname{coth}\left[\frac{\bar{L} \bar{\lambda}_{e}}{4}\right]+\bar{L} \bar{\lambda}_{e} \operatorname{coth}\left[\frac{\bar{L} \bar{\lambda}_{e}}{4}\right]^{2}}{2 \eta \xi},
$$

where, $F$ depends on the materials constants and the boundary conditions.

It should be noted, that according to this model the fragmentation process can be unreally continued because the behavior of the interface is assumed to be ideally elastic.

\section{CONCLUSION}

The phenomenological model describing cracking under uniaxial tensile loading of brittle thin film on a deformable substrate with an elastic interface layer is considered. The presented analytical solution predicts the averaged crack density as a function of applied loading and materials parameters. The layer cracking modes are analyzed by applying the shear-lag approach.

\section{REFERENCES}

[1] K. P. Mróz, A. Bigos, S. Kucharski, K. Dolinski, and E Bełtowska-Lehman, "Ni-W electrodeposited coatings on low carbon steel substrate: Fatigue observations," Journal of Materials Engineering and Performance, vol. 23, pp. 3459-3466, 2014.

[2] M. Białas and Z. Mróz, "Modelling of progressive interface failure under combined normal compression and shear stress," Int. Journal of Solids and Structures, vol. 42, pp. 4436-4467, 2005.

[3] M. Białas and Z. Mróz, "Crack patterns in thin layers under temperature loading. Part I: Monotonic loading," Eng. Fracture Mechanics, vol. 73, pp. 917-938, 2006.

[4] G. Nikolova, J. Ivanova, and Z. Mróz, "Modelling of thermally induced progressive delamination in two-plate structure," J. Theoret. and Applied Mech., vol. 36, pp. 71-92, 2006.

[5] L. H. Cox, "The elasticity and strength of paper and other fibrous materials," Brit. J. Appl. Phys, vol. 3, pp. 72-79, 1952.

[6] A. Kelly and W. R. Tyson, "Ensile properties of fiber-reinforced metals: copper/tungsten and copper/molybdenum," J. Mech. Phys. Solids, vol. 13, pp. 329-350, 1965

[7] J. M. Hedgepeth, "Stress concentrations in filamentary structures," NASA TN D-882, 1961.

[8] J. Ivanova, V. Valeva, and Z. Mróz, "Mechanical modelling of the delamination of bi-material plate structure," J. Theoret. and Applied Mech., vol. 36, pp. 39-54, 2006.

[9] A. P. McGuigan, G. A. D. Briggs, V. M. Burlakov, M. Yanaka, and Y. Tsukahara, "An elastic-plastic shear lag model for fracture of layered coatings," Thin Solid Film, vol. 424, pp. 219-223, 2003.

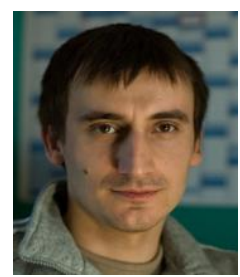

Krzysztof P. Mróz was born in 1977 in Ostrowiec Świetokrzyski, Poland. In 1996 he started his studies at Electrotechnics and Informatics Departments of the Rzeszow University of Technology. Here he has focused on simulation of an electricity grid. Simultaneously in 1998 he began his studies at the Management Department. After his diploma thesis he started the $\mathrm{PhD}$ studies of mechanical engineering at Institute of Fundamental Technological Research, Polish Academy of Science (IPPT PAN). Here he has focused on fatigue and fracture. After his $\mathrm{PhD}$ thesis in 2009 he worked as a researcher at IPPT PAN. His fields of research include fatigue and fracture of advanced materials.

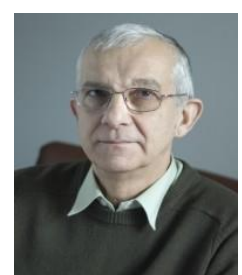

Krzysztof Doliński was born in Lodz (Poland) in 1949. In 1972 he finished his civil engineering studies at Technologica University in Lodz. After his diploma thesis he started the $\mathrm{PhD}$ studies at Institute of Fundamental Technological Research, Polish Academy of Science (IPPT PAN). His PhD thesis earned in 1975 was devoted to stochastic analysis of structures. In 1993 he defended at IPPT PAN his habilitation thesis on stochastic modelling of fatigue. Since 1975 he has worked as a researcher at IPPT PAN. His fields of research include reliability of structures, fatigue and fracture of advanced materials.

In 1981-1983 he worked at Technical University of Munich as an Alexander von Humboldt fellow and in 1984-1985 worked there as a Volkswagen-Werke fellow. He visited the Universities in Germany, Italy and Sweden as a visiting professor. He published more than 50 scientific papers and more than 70 citations of his works could be find in ISI. 